\title{
Analysis of Vibration and Sound Radiation Characteristics of Resilient Wheel in Metro
}

\author{
Jianhui Tian*, Ke Wang, Kan Xiao \\ CAE Analysis Room for Engineering Application, School of Mechatronic Engineering, Xi'an Technological University, Xi'an, China \\ Email address: \\ 540673737@qq.com (Jianhui Tian) \\ ${ }^{*}$ Corresponding author \\ To cite this article: \\ Jianhui Tian, Ke Wang, Kan Xiao. Analysis of Vibration and Sound Radiation Characteristics of Resilient Wheel in Metro. American Journal \\ of Mechanical and Industrial Engineering. Vol. 3, No. 4, 2018, pp. 55-63. doi: 10.11648/j.ajmie.20180304.13
}

Received: July 17, 2018; Accepted: August 6, 2018; Published: September 15, 2018

\begin{abstract}
The wheel of vehicles is not pure rolling on the rail for the track irregularity, but with a small vertical and horizontal relative displacement. The relative displacement produces the wheel rail force and causes the vibration of the wheel and rail, which is transmitted to the air and produces noise. Therefore, it is of great significance to study the wheel's vibration and sound radiation under the track irregularity conditions. In this paper, the wheel-rail coupling dynamic analysis model of metro vehicle was established by using UM software and was researched by numerical calculation method, the wheel-rail contact force under irregularity excitation was obtained. Then the acoustic calculation model of the rigid wheel and the resilient wheel was established. Combined with the structural finite element method and the acoustic boundary element method, the structural response obtained by transient dynamic analysis was used as the boundary condition to study the acoustic radiation characteristics and the noise reduction effect of the resilient wheel. The study results show that under the vertical excitation of wheel-rail, compared with rigid wheel, the use of resilient wheel can inhibit the vibration for rim, tread and web. The vibration acceleration attenuation of the web is most significant, from $97 \mathrm{~m} / \mathrm{s} 2$ to $58 \mathrm{~m} / \mathrm{s} 2$. It is because of the vibration reduction function of the rubber body for the resilient wheel. The sound power level of the resilient wheel has a slight increase in the low frequency range and has a slight decrease in the high frequency range, in the whole calculated frequency range the sound power level of the resilient wheel decreases by $10.1 \mathrm{~dB}$. These provide a good reference for the resilient wheel application in metro vehicle.
\end{abstract}

Keywords: Resilient Wheel, Track Irregularity, Dynamic, Vibration, Sound Radiation

\section{Introduction}

Rail vehicle has become one of the most effective means to alleviate urban congestion by virtue of its strong passenger transport capacity and convenience. However, the vibration and noise generated by the vehicle operation not only reduce the ride comfort of the vehicle, but also increase the operating cost [1]. It has become one of the important factors restricting the development of rail transit.

It is generally believed that the wheel-rail contact force caused by track irregularity and special operating conditions causes the structural vibration of the wheel structure and track structure, which propagates in the air to produce wheel-rail noise [2]. According to the causes of wheel-rail force, wheel-rail noise can be divided into rolling noise, impact noise and curve whistling noise. Among them, impact noise and curve whistling noise can be controlled by increasing the turning radius of track line and using seamless rail [3]. However, rolling noise always exists with the running of vehicle, so controlling rolling noise is the key to control wheel-rail noise. From the Remington rolling noise prediction model, it can be seen that the wheel is one of the main noise contributors. Therefore, resilient wheel as a relatively good vibration and noise reduction measure is used in rail vehicles [4]. Different types of noise occupy a dominant role in different speed ranges. When the train running speed is between $35 \mathrm{~km} / \mathrm{h}$ and $250 \mathrm{~km} / \mathrm{h}$, the noise of the train mainly appears as wheel-rail noise, so the noise of urban rail vehicles mainly appears as wheel-rail noise [5]. The noise test for elastic wheels in Hamburg Metro, Germany shows that the $20 \sim 22 \mathrm{~dB}$ of metro vehicles with resilient wheel can be reduced in the curve segment [6]. The Modal damping ratio of rigid Wheels is between 0.0001 and 0.0003 , Remington thinks that the noise reduction effect is related to the modal loss 
factor of the wheel, and if the Modal damping ratio exceeds 0.007 , it can effectively suppress curve whistling noise [7]. Jones compares the rolling noise of the rigid wheel and the resilient wheel by using the TWINS model, and find that the noise of the resilient wheel is reduced by $7 \mathrm{~dB}[8]$. Zhao et al. test the noise of resilient wheel by hammering method, and concluded that the amplitude-frequency characteristics of the noise were improved very well by the resilient wheel. [9]. Liu et al. study the effect of rubber properties on the vibration and noise reduction of resilient wheel, and find that increasing the elastic modulus and damping loss factor of rubber material are beneficial to reduce the vibration and noise of wheel [10]. Wen et al. establish the vehicle-track coupling system model of resilient wheel and study the vehicle running stationary and curve passing, and find that the radial and axial stiffness of elastic elements can be matched reasonably to obtain better dynamic performance [11].

In this study, firstly, the wheel-rail coupling dynamic model of metro vehicle is established. Then the wheel-rail force considering track irregularity is calculated on the basis of hertz's nonlinear contact theory. Lastly, the hybrid numerical simulation model of the finite element and boundary element for resilient wheel is established to obtain the sound power level.

\section{Wheel-Rail Contact Force of Metro Vehicle}

\subsection{Wheel-Rail Coupling Dynamics Model for Metro Vehicle}

Because of the irregularity between the wheel and rail, when the train travels on the rail at a certain speed, the wheel-rail interaction will produce the wheel-rail contact force. On the one hand, the wheel-rail contact force causes the vibration of the rail and the wheel. On the other hand, the vibration along the axle is transmitted to the body through double suspension systems attenuation, which causes the vibration of the structure. In the multi-rigid vehicle dynamics model, the vehicle can be simplified into three parts, which are carbody, frame and wheelset. They are connected by the suspension, and the suspension system can be regarded as a spring with damping and stiffness. The wheel-rail contact model of the vehicle with double suspension systems is shown in figure 1 .

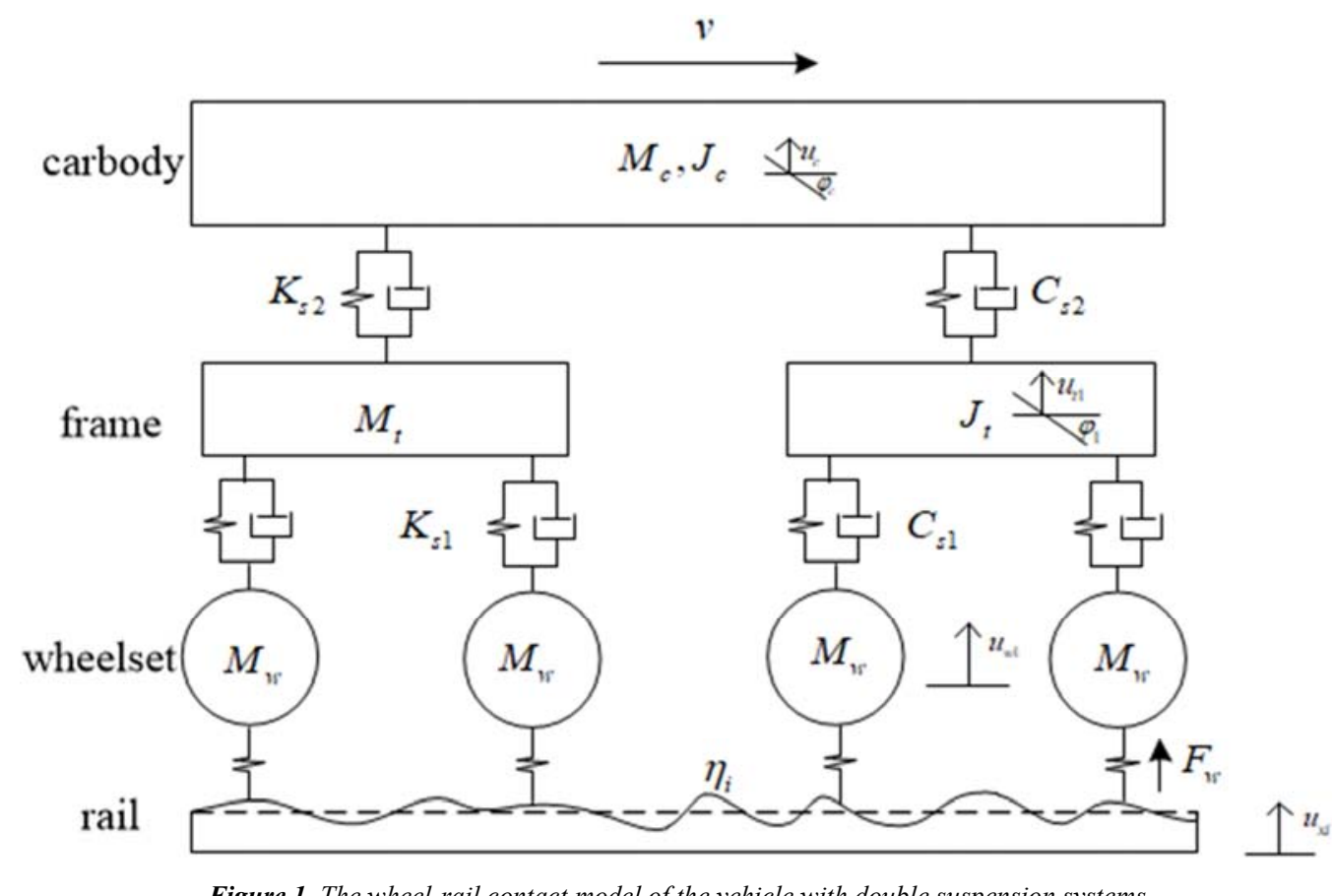

Figure 1. The wheel-rail contact model of the vehicle with double suspension systems.

In figure $1, M_{w}, M_{t}, M_{c}$ represent the mass of the wheel, the frame and the carbody, respectively; $J_{t}, J_{c}$ represent the moment of inertia of frame and carbody, respectively; $K_{s 1}$, $K_{s 2}$ represent the vertical stiffness of primary suspension and secondary suspension, respectively; $C_{s 1}, C_{s 2}$ represent the vertical damping of primary suspension and secondary suspension, respectively; $u_{x i}, u_{w 1}, u_{t 1}, u_{c}$ represent the vertical displacement of the rail, the wheelset, the frame and the carbody, respectively; $\varphi_{1}, \varphi_{c}$ represent the pitching angle of frame and carbody, respectively; $v$ represent the vehicle speed.

In this model, the vertical and pitching directions motion of carbody and frame are considered, but only the vertical direction motion of the wheelset is considered. The vibration equation of the vehicle under the action of track irregularity is as follows [12].

$$
M \ddot{u}+C \dot{u}+K u=Q+F_{w}
$$

where $M$ is the inertia matrix; $C$ is the damping matrix 
caused by primary suspension and secondary suspension; $K$ is the stiffness matrix caused by primary suspension and secondary suspension; $u$ is the vertical displacement matrix for car body, frame and wheelset; $\dot{u}$ is the vertical velocity matrix for car body, frame and wheelset; $\ddot{u}$ is the vertical acceleraction matrix for car body, frame and wheelset; $Q$ is the vehicle gravity vector; $F_{w}$ is the wheel-rail contact force vector.

The wheel-rail contact force mainly comes from the vertical direction when the vehicle is traveling in a uniform speed straight line. According to the wheel-rail coupling rigid multi-body dynamics model, the wheel-rail contact force can be obtained from Hertz's nonlinear elastic contact theory by using the relative vertical displacement of wheel-rail contact.

The wheel-rail contact force is expressed as follows:

$$
F_{w i}=\left\{\begin{array}{l}
\frac{1}{G^{3 / 2}}\left(\left|u_{w i}-\left(u_{x i}+\eta_{i}\right)\right|\right)^{3 / 2}, u_{w i}-\left(u_{x i}+\eta_{i}\right) \leq 0 \\
0, u_{w i}-\left(u_{x i}+\eta_{i}\right)>0
\end{array}\right.
$$

where $F_{w i}$ is the contact force of the $i$ th wheel of the vehicle; $u_{w i}$ and $u_{x i}$ are the displacement of wheel and rail in the coordinate point $x_{i}$, respectively; $\eta_{i}$ is the time-frequency domain sample of track irregularity in the the coordinate point $x_{i} ; G$ is the contact deflection coefficient.

Suppose the irregularity sample of the rail is as follows:

$$
\eta_{i}=\eta\left(x_{i}\right)
$$

where $\eta\left(x_{i}\right)$ is the irregularity value in the coordinate point $x_{i}$, which is time-frequency domain samples simulated by the track irregularity PSD (power spectral density).

\subsection{Track Irregularity}

Track irregularity is a complex stochastic process, which can be simulated as a random function varying with the length of the rail. PSD is the most commonly used statistical function to describe track irregularity and stationary stochastic process, which is fitted with spatial frequency.

American fifth grade track irregularity PSD is widely used in the vehicle dynamics analysis of metro vehicles, but the wheel-rail forces studied in this paper need to be wrapped in a high frequency component that can arouse the high frequency vibration of the wheels. Therefore, the time-frequency domain samples of track irregularity is $\eta$, which is obtained by combining American's track irregularity PSD for fifth grade and Sato's track irregularity PSD in this paper.

The vertical irregularity of American's track irregularity

$$
|X(k)|=|D F T[x(n)]|=N \sqrt{S_{x}(f=k \cdot \Delta f) \Delta f} \quad(k=0,1, \cdots, N-1)
$$

Cause $x(n)$ is a stochastic process, so assuming that its independent phase sequence is $\xi_{k}$, with $\left|\xi_{k}\right|=1$.
PSD for fifth grade is expressed as follows:

$$
S_{v}(\omega)=\frac{k A_{v} \omega_{c}^{2}}{\omega^{2}\left(\omega^{2}+\omega_{c}^{2}\right)}\left(\mathrm{cm}^{2} / \mathrm{rad} / \mathrm{m}\right)
$$

where $\omega$ is the spatial frequency; $\omega_{c}$ is the truncation frequency, $\omega_{c}=0.8245 \mathrm{rad} / \mathrm{m} ; A_{v}$ is the roughness coefficient, $\quad A_{v}=0.2095 \mathrm{~cm}^{2} \cdot \mathrm{rad} / \mathrm{m} ; k$ is constant coefficient, $k=0.25$.

The Sato's track irregularity PSD is expressed as follows

$$
S(\omega)=\frac{A}{\omega^{3}}\left(\mathrm{~m}^{2} / \mathrm{rad} / \mathrm{m}\right)
$$

where $A$ is the roughness coefficient, $A=4.15 \times 10^{-8} \sim 5.0 \times 10^{-7}$. This PSD is widely used as the excitation source in the prediction of wheel-rail high frequency vibration and noise.

The track irregularity PSD can be directly used as the excitation input only when it is converted into time domain spectrum. So use the the Blackman-Turkey method to estimate the PSD, the following result can be obtained [13].

$$
S(k)=\frac{|D F T[\mathrm{x}(n)]|^{2}}{N^{2}}=\frac{|X(k)|^{2}}{N^{2}} \quad(k=0,1, \cdots, N-1)
$$

where $S(k) \quad k=0,1, \cdots, N-1$ is PSD; DFT is discrete fourier transform; $X(k)$ is spectrum of the time series $x(n)$ $n=0,1, \cdots, N-1 ; k$ is sampling interval number; $N$ is the total sampling number.

According to the conservation of energy.

$$
S(\omega) d \omega=S(f) d f=S(k) d k
$$

where $S(f)$ is the PSD in time-frequency domain.

The relationship between $f$ and $k$ is as follows.

$$
f=k \cdot \Delta f \quad(k=0,1, \cdots, N-1)
$$

where $\Delta f$ is the frequency sampling interval.

Therefore, according to the formula (7) and (8), we can get the following formula.

$$
S(k)=S(f) \cdot \Delta f
$$

So, the modular value of $X(k)$ can be obtained by combining the formulas (6) and (9) as follows. 
Then the $X(k)$ is transformed by FFT, and the time series of track irregularity PSD can be obtained as follows.

$$
x(n)=\frac{1}{N} \sum_{k=0}^{N-1} X(k) e^{i 2 \pi k n / N} \quad(n=0,1, \cdots, N-1)
$$

According to the formula (4) (12), the time domain samples of the American's track irregularity PSD for grade five and the Sato's track irregularity PSD are $x_{a}(n)$ and $x_{s}(n)$ respectively. So the time domain samples of track irregularity can be obtained as follows:

$$
\eta=x_{a}(n)+x_{s}(n) \quad(n=0,1, \cdots, N-1)
$$

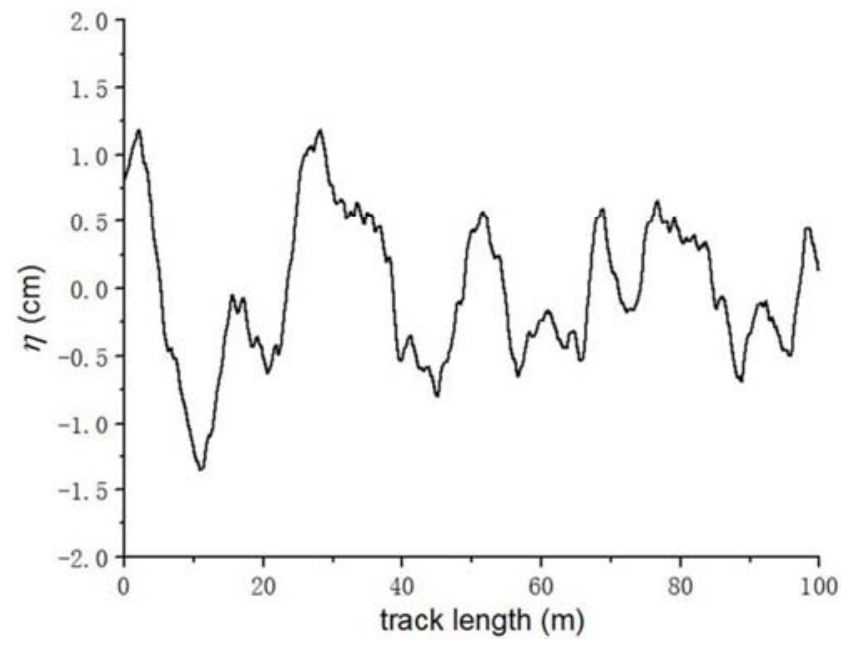

Figure 2. The time domain samples of track irregularity.

\subsection{Simulation Model of Metro Vehicle and Wheel-Rail Vertical Force}

This paper takes B-type metro vehicle as the research object, based on the multi-body dynamics theory, the vehicle simulation model is established by using the multi-body dynamics software Universal Mechanism (UM). The vehicle is simplified into one carbody, two frames and two wheelset. The nonlinear wheel-rail contact relationship and the suspension nonlinearity are also taken into account. The partial vehicle parameters are shown in the table 1 [14].

Table 1. The partial vehicle parameters.

\begin{tabular}{ll}
\hline Model Parameters & Values \\
\hline Quality of frame & $1500 \mathrm{~kg}$ \\
Quality of carbody & $44800 \mathrm{~kg}$ \\
quality of wheelset & $1400 \mathrm{~kg}$ \\
The vertical stiffness of primary suspension & $0.71 \mathrm{MN} / \mathrm{m}$ \\
The lateral stiffness of primary suspension & $3.18 \mathrm{MN} / \mathrm{m}$ \\
The longitudinal stiffness of primary suspension & $4.79 \mathrm{MN} / \mathrm{m}$ \\
The vertical stiffness of secondary suspension & $0.45 \mathrm{MN} / \mathrm{m}$ \\
The lateral stiffness of secondary suspension & $0.20 \mathrm{MN} / \mathrm{m}$ \\
The longitudinal stiffness of secondary suspension & $0.20 \mathrm{MN} / \mathrm{m}$ \\
The vertical damping of primary suspension & $20000 \mathrm{~N} \cdot \mathrm{s} / \mathrm{m}$ \\
The vertical damping of secondary suspension & $60000 \mathrm{~N} \cdot \mathrm{S} / \mathrm{m}$ \\
\hline
\end{tabular}

The vehicle simulation model and the rail is established by UM software, and the wheel-rail coupling model of metro vehicle is shown in the figure 3 . The track irregularity time domain samples obtained by numerical simulation are used to solve wheel-rail contact force. The time history curve of vertical wheel-rail forces is extracted while the vehicle is traveling at a constant speed of $80 \mathrm{~km} / \mathrm{h}$. The time history curve of the wheel-rail force is shown in figure 4.

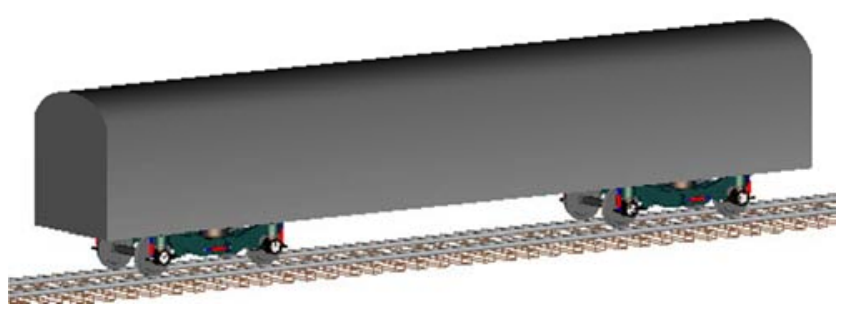

Figure 3. Wheel-rail coupling model of metro vehicle.

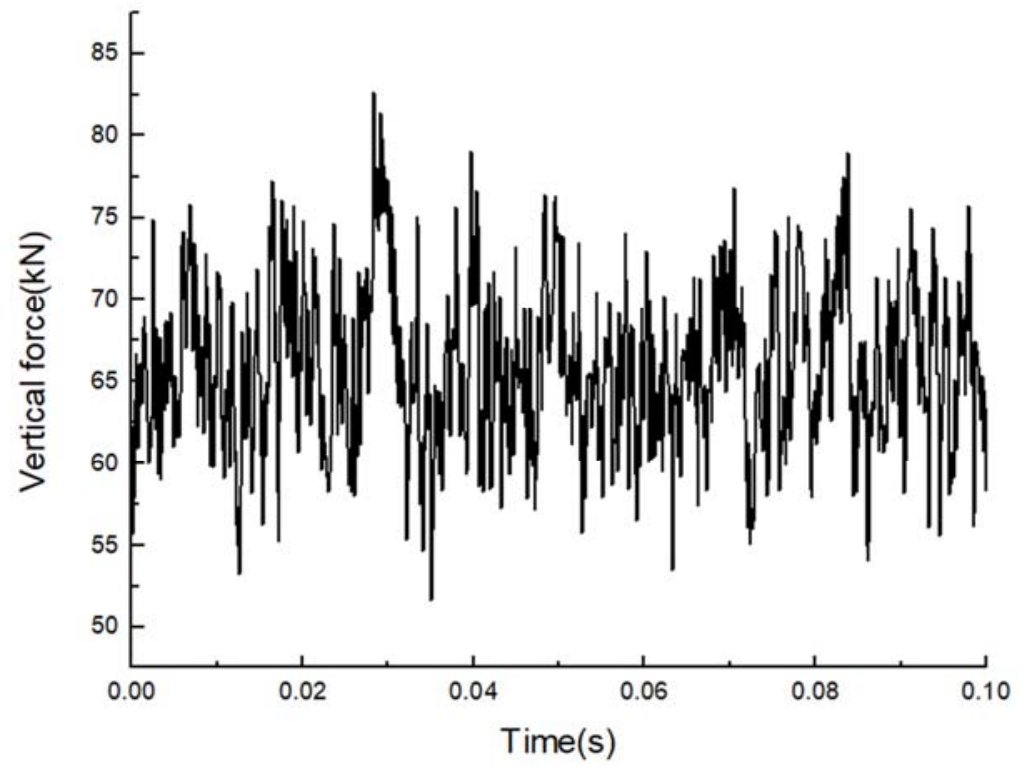

Figure 4. The vertical wheel-rail force. 


\section{Vibration Analysis}

\subsection{Calculation Model}

The rubber used in the elastic layer of resilient wheel is a nonlinear material with the characteristics of large deformation and incompressible volume. Any nonlinear (such as plastic and contact element), even if defined, will be ignored, so that the rubber material is linearized in the finite element simulation of the resilient wheel, The young's modulus is $2300 \mathrm{MPa}$, poisson's ratio is 0.49 , density is $1050 \mathrm{~kg} / \mathrm{m}^{3}$ and the damping loss factor of rubber material is 0.1 , respectively. At the same time, the finite element model of the rigid wheel and the resilient wheel of the metro are established. The elastic layer of the resilient wheel is discretized by solid185, the other parts of the resilient wheel are discretized by the solid45. The resilient wheel is eventually discretized into 31988 elements and 39843 nodes. The rigid wheel is eventually discretized into 17673 elements and 23349 nodes. The material constants of steel for wheel are as follows: young's modulus is $2.1 \mathrm{e} 5 \mathrm{MPa}$, poisson's ratio is 0.3 , density is $7800 \mathrm{~kg} / \mathrm{m}^{3}$. The finite element mesh of rigid wheel and resilient wheel section are shown in figure 5 and figure 6.

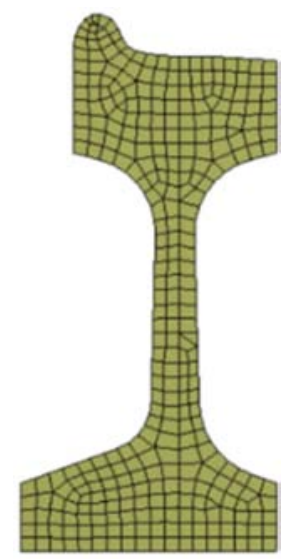

Figure 5. The finite element mesh of rigid wheel section.

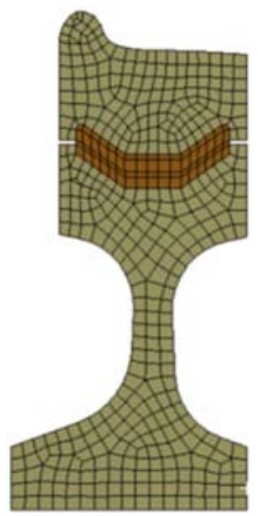

Figure 6. The finite element mesh of resilient wheel section.

A cylindrical coordinate system is established for the inner surface of the hub hole as a reference, and axial translation constraints and circumferential rotation constraints are applied to all nodes in the surface of the hub hole.

\subsection{Transient Dynamic Analysis}

In transient dynamic analysis, the vibration of wheel is studied with wheel-rail vertical force as excitation. The wheel-rail vertical force is applied to the wheel-rail nominal contact point in y negative direction. At the same time, the acceleration response of the tread, rim and web are observed in y negative direction as shown in figure 7.

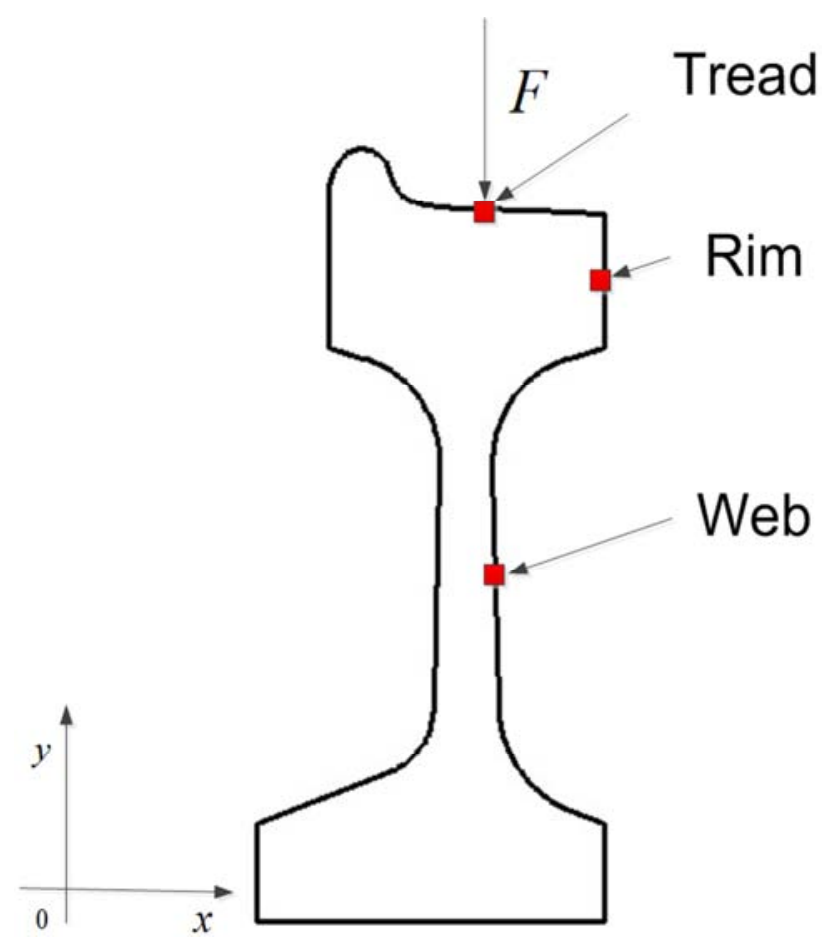

Figure 7. The loading position and the acceleration response observation position.

The acceleration response of the tread, rim, and web of the rigid wheel and the resilient wheel is obtained using finite element method in figure 8 and figure 9 , respectively.

It can be seen from figure 8 and figure 9 that the vibration acceleration of wheel tread is larger than that of rim and web, regardless of whether it is resilient wheel or rigid wheel. Through comparison, it is found that the vibration trend of rigid wheel tread, rim and web is close, and the resilient wheel has obvious restraining effect for radial vibration of web. The web's radial maximum radial vibration acceleration of the rigid wheel is $97 \mathrm{~m} / \mathrm{s}^{2}$, and the maximum radial vibration acceleration of the resilient wheel is $58 \mathrm{~m} / \mathrm{s}^{2}$. That is to say, the vibration reduction effect of the tread and rim is not obvious, the vibration of the web is significantly reduced because of the vibration reduction function of the rubber body for the resilient wheel. The maximum acceleration response of each part of the wheel is shown in table 2 . 


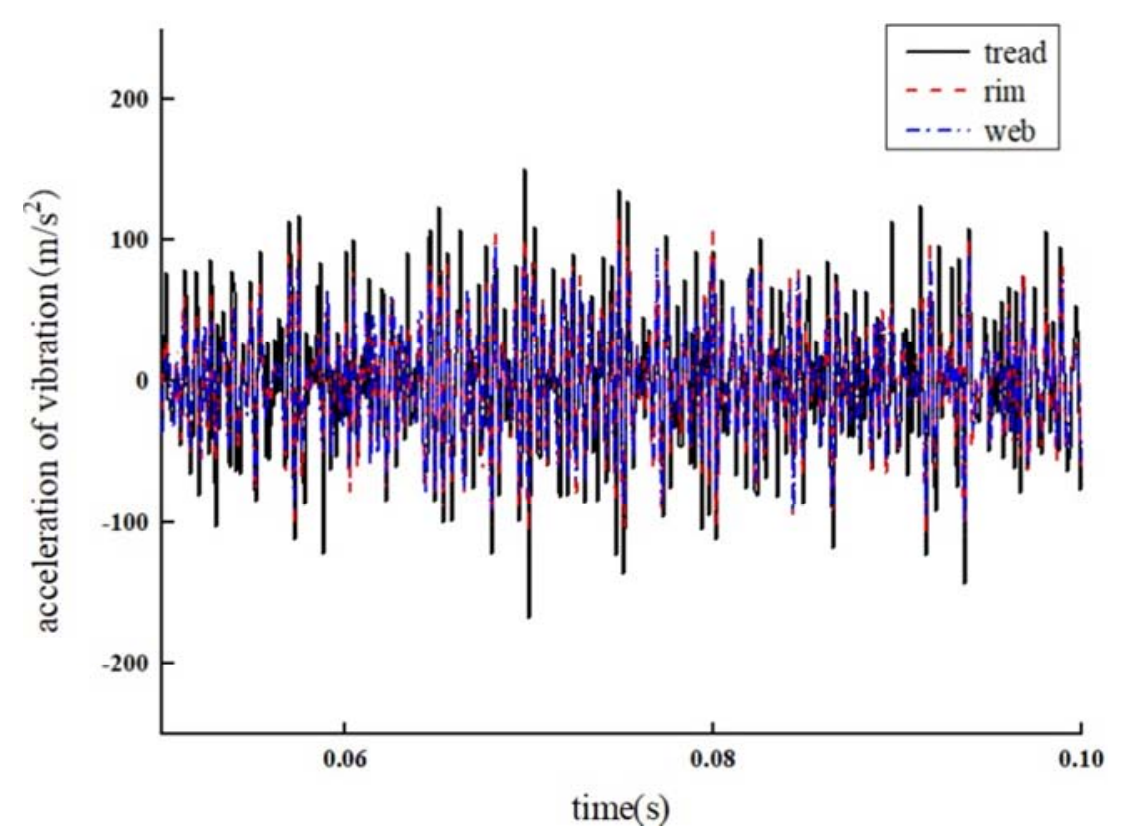

Figure 8. Acceleration response of the rigid wheel.

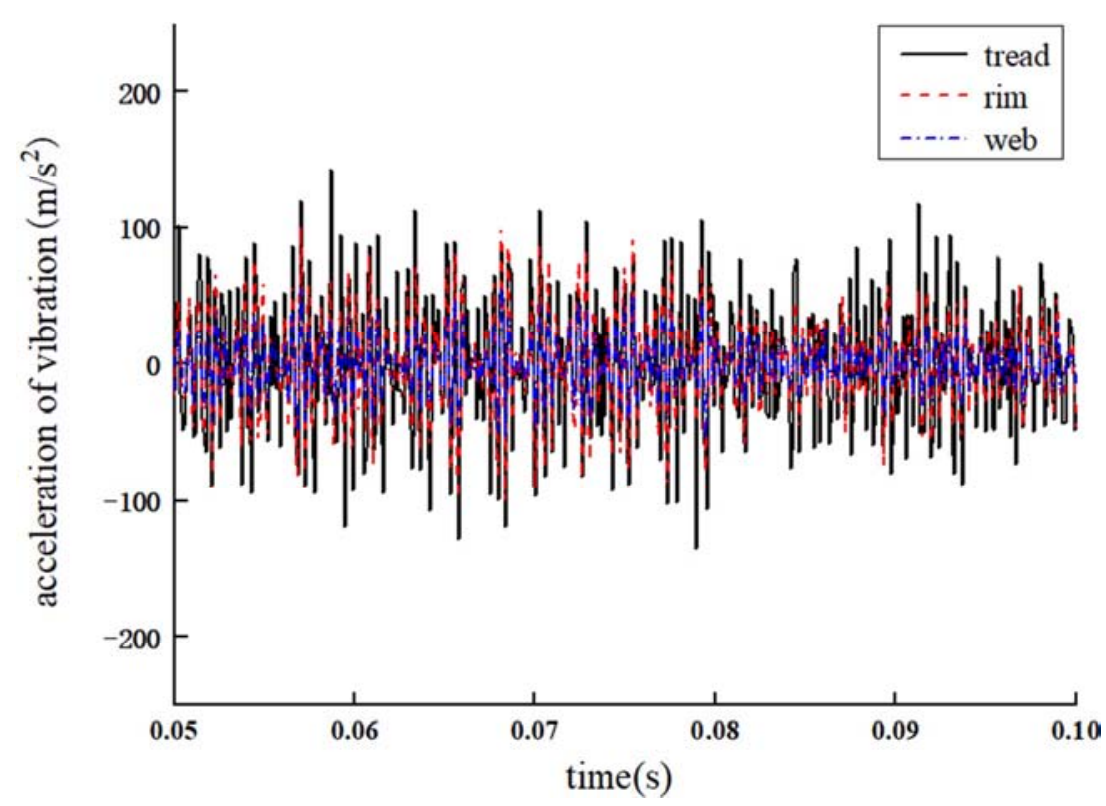

Figure 9. Acceleration responses of the resilient wheel.

Table 2. The maximum acceleration response of each part of the wheel.

\begin{tabular}{lll}
\hline parts & rigid wheel $\left(\mathbf{m} / \mathbf{s}^{2}\right)$ & resilient wheel $\left(\mathbf{m} / \mathbf{s}^{2}\right)$ \\
\hline tread & 151 & 143 \\
rim & 116 & 101 \\
web & 97 & 58 \\
\hline
\end{tabular}

\section{Sound Radiation}

\subsection{Boundary Element Model}

The following principles are generally followed in the establishment of acoustic boundary element models:

$$
f_{\max }=\frac{c}{6 L}
$$

In the formula, $f_{\max }$ is the highest frequency calculated, $c$ is the sound velocity, $L$ is the element length. If the maximum frequency of acoustic calculation is specified, the maximum element length must be satisfied as following.

$$
L \leq \frac{c}{6 f_{\max }}
$$

Therefore, in order to reduce the solving time, the frequency range, step size and mesh size of the sound calculation of resilient wheel are determined to be $40 \sim 4000 \mathrm{~Hz}$, $40 \mathrm{~Hz}$ and $13 \mathrm{~mm}$, respectively, on the basis of ensuring that the calculation covers the main acoustic energy radiation frequency. 


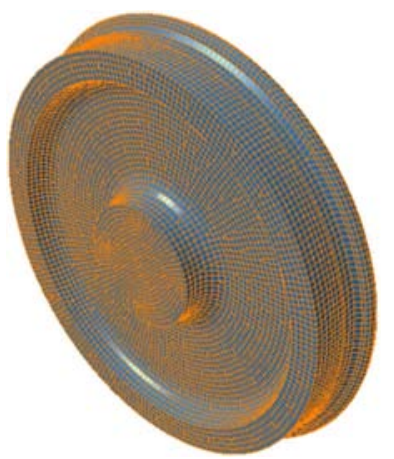

In order to prevent the sound energy leakage from the hub hole, the hub hole is closed by shell element. The calculated fluid medium is air, the air density $\rho$ is $1.225 \mathrm{~kg} / \mathrm{m}^{3}$, and the sound velocity in the air is $340 \mathrm{~m} / \mathrm{s}$. The resilient wheel boundary element model is shown in figure 10 .

\subsection{Sound Power Level Analysis}

By sound analysis of rigid wheel and resilient wheel, the spectrum of equal bandwidth sound power level of two kind wheel is shown in figure 11.

Figure 10. The resilient wheel boundary element model.

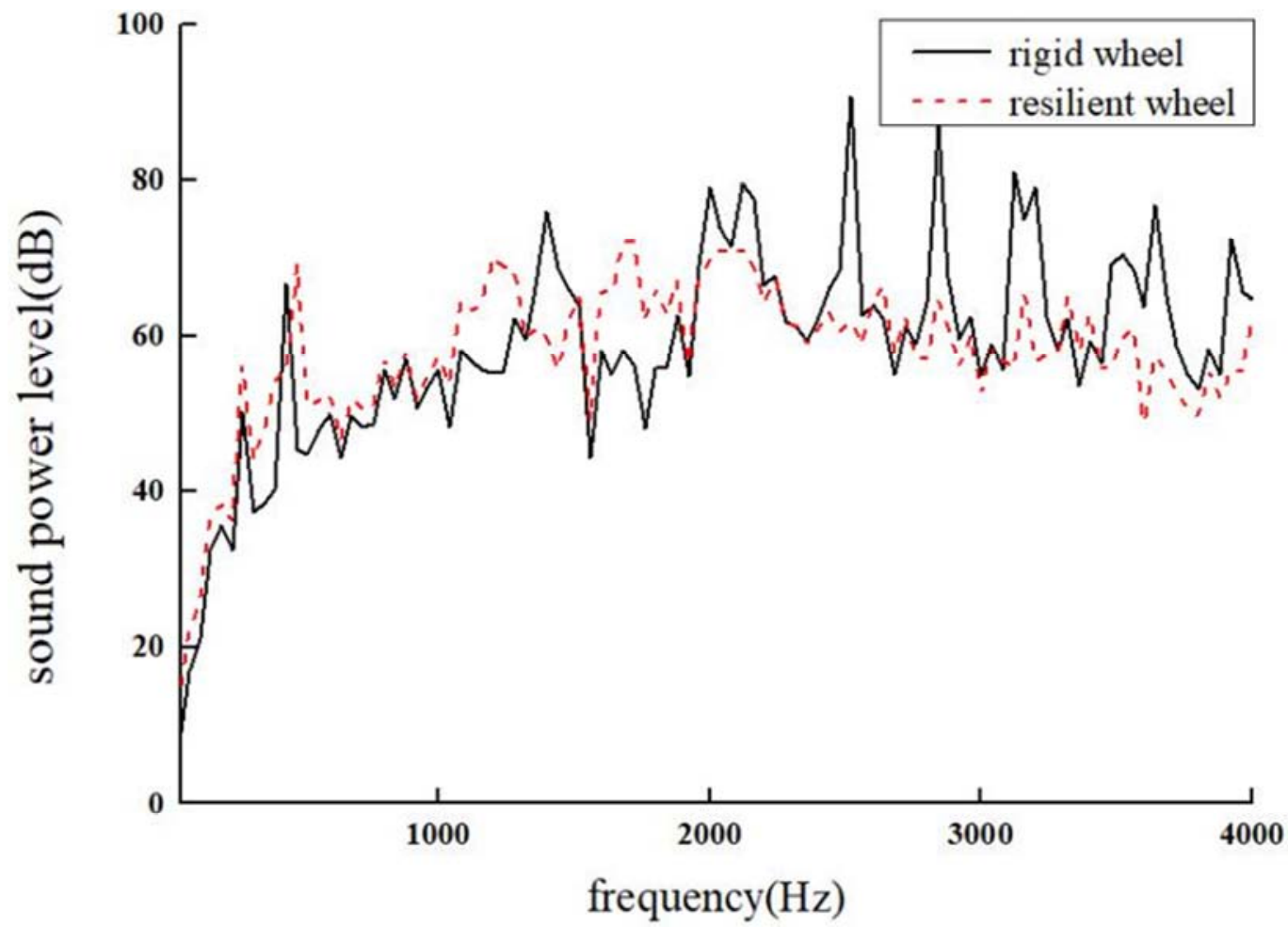

Figure 11. The equal bandwidth sound power level.

It can be seen from figure 11 that the sound radiation of the wheel is mainly concentrated in medium and high frequency range, regardless of whether it is rigid wheel or resilient wheel. It can be found that the sound radiation of resilient wheel is slightly higher than that of the rigid wheel in the low frequency band, and decreased in the medium and high frequency band. Compared with rigid wheel, under vertical wheel-rail force excitation, the sound power level of the resilient wheel decreases by $15 \mathrm{~dB}, 9.4 \mathrm{~dB}, 28.7 \mathrm{~dB}, 22.3 \mathrm{~dB}$, $25.2 \mathrm{~dB}, 19.5 \mathrm{~dB}$ at the frequency $1400 \mathrm{~Hz}, 2520 \mathrm{~Hz}, 2840 \mathrm{~Hz}$, $3120 \mathrm{~Hz}$ and $3640 \mathrm{~Hz}$, respectively.

In order to further understand the spectrum characteristics of rigid wheel and resilient wheel, the spectrum of equal bandwidth sound power level is converted to the $1 / 3$ octave sound power level as shown in figure 12 .

Before the band of $1000 \mathrm{~Hz}$, the sound power level of the resilient wheel increased slightly. After the band of $1000 \mathrm{~Hz}$, the sound radiation effect of the resilient wheel is obvious, which is reduced by $5.69 \mathrm{~dB}, 17.14 \mathrm{~dB}, 15.78 \mathrm{~dB}$ and 12.08 $\mathrm{dB}$ at the frequency band of $2000 \mathrm{~Hz}, 2500 \mathrm{~Hz}, 3150 \mathrm{~Hz}$ and $4000 \mathrm{~Hz}$, respectively. Compared with rigid wheel, although the sound radiation of the resilient wheel increases slightly in the low frequency range, the sound radiation reduction effect is remarkable in the medium and high frequency range, the sound radiation reduction is $1.6 \mathrm{~dB}$ within $2000 \mathrm{~Hz}$ and $10.1 \mathrm{~dB}$ within $4000 \mathrm{~Hz}$, which indicates that the sound radiation reduction effect of resilient wheel is great. 


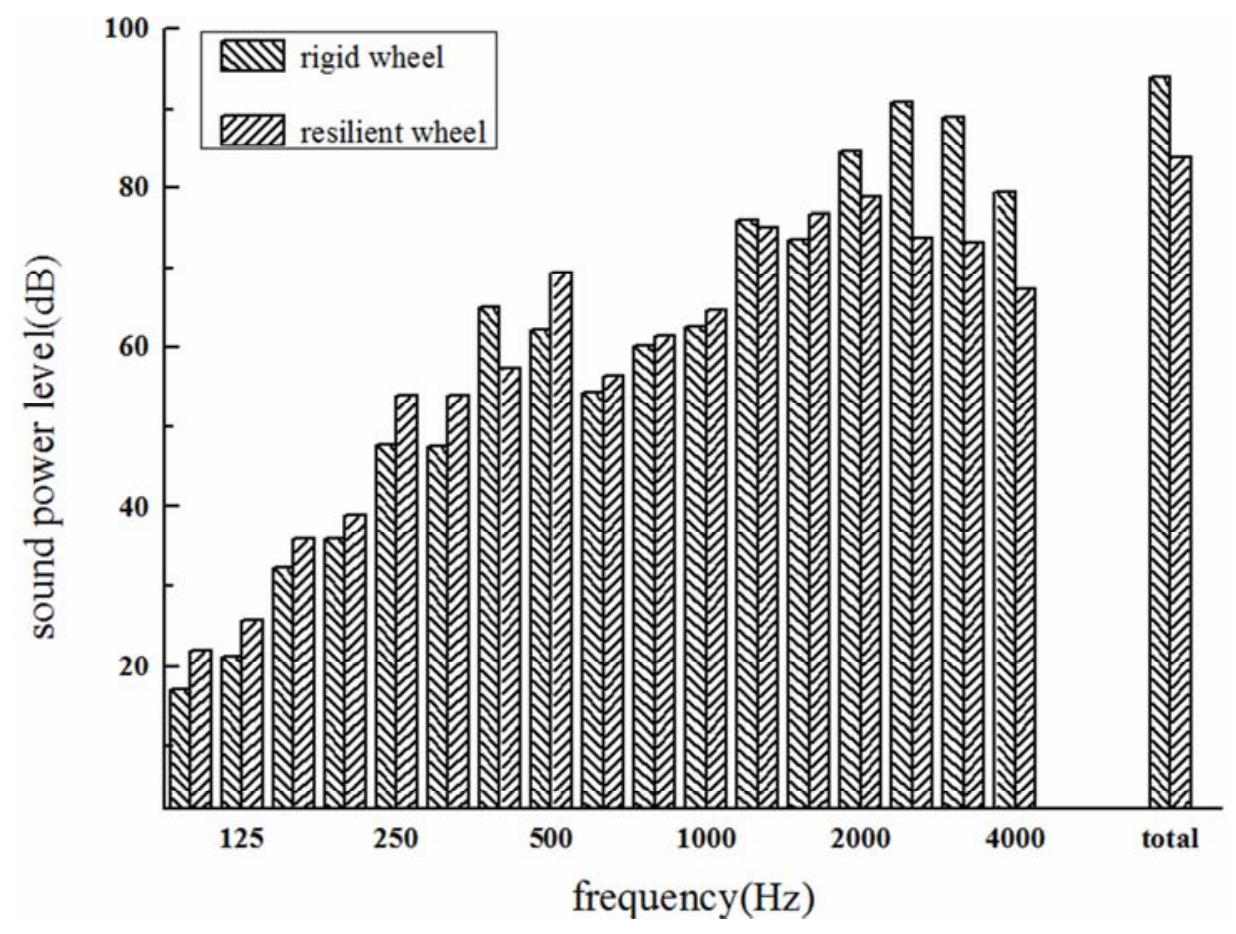

Figure 12. 1/3 octave sound power level.

\section{Conclusions}

In this paper, the track irregularity is numerically simulated, and the dynamic model of wheel-rail coupling system is established, and the wheel-rail contact force is obtained. Then, the vibration reduction and sound radiation of resilient wheels are studied by using finite element method and boundary element method, and the following conclusions are obtained.

(1) Under the vertical excitation of wheel-rail, compared with rigid wheel, the use of resilient wheel can inhibit vibration for rim, tread and web. The radial maximum acceleration of the web of the rigid wheel and the resilient wheel is $97 \mathrm{~m} / \mathrm{s}^{2}$ and $58 \mathrm{~m} / \mathrm{s}^{2}$ respectively, which indicates that the vibration of the web is significantly reduced because of the vibration reduction function of the rubber body for the resilient wheel.

(2) Both the rigid wheel and the resilient wheel have low acoustic radiation efficiency in the low frequency range, indicating that the radiation acoustic energy of the wheel is mainly concentrated in the middle and high frequency range. Compared with the rigid wheel, the acoustic radiation efficiency of resilient wheel is basically positively correlated with the frequency within $1000 \mathrm{~Hz}$, and its acoustic radiation efficiency is higher within $1000 \mathrm{~Hz}$. The sound radiation reduction effect is remarkable in the middle and high frequency range, and the sound radiation reduction is $10.1 \mathrm{~dB}$ within $4000 \mathrm{~Hz}$.

\section{Acknowledgements}

The research is supported by the National Natural Science Foundation of China (No.11302159), Basic Research Plan of Natural Science in Shannxi Province (No.2018JM5099), and the Principal Foundation of Xi'an Technological University (No. XAGDXJJ18004).

\section{References}

[1] Tian JH, Jian L, Li B (2016) Investigation of critical limit value of subway complaint noise. Noise and Vibration Control 36:106-108.

[2] Xu ZS, Zhai WM (2005) Prediction model of wheel-rail noise for rail transit. Journal of Traffic and Transportation Engineering 5:14-18.

[3] Lu YR (2010) Research and application of continuously welded rail trac. China Railway Publishing House, Bei Jing.

[4] Ma YX, Liu CS (2017) Prediction model of wheel-rail noise for rail transit. Science and Technology \& Innovation 1:36-41.

[5] Han J, Xiao XB (2012) Sound radiation characteristics of wheels used in urban rail traffic. Journal of Mechanical Engineering 48:115-121.

[6] Wang BM, Zhao Q (1998) The elastic gum wheels in lrt vehicles. Urban Mass Transit 1: 37-40.

[7] Remington PJ (1988) Wheel/rail rolling noise: what do we know? what don't we know? where do we go from here? Journal of Sound \& Vibration 120:203-226.

[8] Jones CJC (2000) Rolling noise generated by railway wheels with visco-elastic layers. Journal of Sound \& Vibration 231:779-790.

[9] Zhao HL, Xu XQ (2001) Study on noise reduction properties of resilient wheels. Journal of the China Railway Society 23:26-30.

[10] Liu YX, Han J, Zhou X (2015) Analysis of Vibration and Noise Reduction Characteristics of Resilient Wheel. Journal of the China Railway Society 37:48-53. 
[11] Wen J, Li F, Yang Y (2015) Parameters optimization and track adaptation research of urban vehicle resilient wheels. Electric Drive for Locomotives 3:74-77.

[12] Lei, XY (2015) High speed railway track dynamics model, algorithm and application. Science Press, Bei Jing.
[13] Liu YH, Li F, Huang YH (2006) Numerical simulation methods of railway track irregularities. Journal of Traffic and Transportation Engineering 6:29-33.

[14] Yan JM, Fu MH (2008) Vehicle engineering. China Railway Publishing House, Bei Jing. 